\title{
Distance Information based Energy Control Mechanisms in the Mobile Sensor Network
}

\author{
Junhyoung Kim, Jeonghyeon Park and Sungkeun Lee ${ }^{1}$ \\ Department of Multimedia Engineering, Sunchon National University, Korea \\ 315 Maegok-dong Suncheon, Jeollanam-do, Korea \\ kjh1570@hanmail.net,pjh6364@nate.com, sklee@sunchon.ac.kr
}

\begin{abstract}
In addition to fixed sensor network such as crop cultivation management system, studies on mobile sensor network have been actively conducting, which is used for collecting information from the moving objects like livestock and cars with use of sensors. The mobility of node enables to prolong the lifespan of network and to manage network efficiently through equal allocation of energy among nodes. To ensure efficient mobility, it is required to make a functional change to MAC layer and routing protocol. In particular, an enhanced transmission mechanism is essential in order to effectively control a link discontinuity or an increase of traffic overhead caused by the mobility of node. This paper aims to propose an energy-efficient transmission mechanism using distance information between sensors in mobile sensor network. Performance of the proposed mechanism has been evaluated by simulation. The simulation results indicate that the proposed mechanism outperforms traditional minimum hop routing method from the perspective of several performance indicators including average packet latency, average energy consumption and network lifespan.
\end{abstract}

Keywords: Mobile sensor network, Energy efficient transmission mechanism, Node mobility, Min hop routing algorithm

\section{Introduction}

Today, massive data as video and images can be sent and received in wireless communication environment. As internet of things became realized due to the development of various sensors, small-scale as well as large-scale wireless network environment is growing rapidly as well. Unlike fixed sensor network as cropping management system, mobile wireless sensor network environment of attaching sensors on people, livestock, and automobile is being investigated in various ways. Because floating node exists in the mobile sensor network, a distant node within a radius of communication and adjacent node can be classified as distance between each node is variable. Due to the nature of wireless communication of communicating by filling air with waves, the output energy consumption according to the output distance increases exponentially. Therefore, unlike existing method of decreasing hop to reduce the consumed energy when exchanging data, this study suggests a method of managing energy efficiently by controlling the output energy according to the distance of the next node. It can be used effectively on mobile wireless sensor network because the number of nodes that pass through increases but the transmission energy decreases. The composition of this study is as follows. The routing technique and adjustment of output energy according to the distance will be explained in Chapter 2. In Chapter 3, this study will make performance evaluation of the suggested mechanism and analyze the simulation results, and make conclusion in Chapter 4.

${ }^{1}$ Corresponding author 


\section{Energy Efficient Routing Mechanism Considering the Distance}

All printed material, including text, illustrations, and charts, must be kept within the parameters of the $815 / 16$-inch (53.75 picas) column length and $515 / 16$-inch (36 picas) column width. Please do not write or print outside of the column parameters. Margins are $3.3 \mathrm{~cm}$ on the left side, $3.65 \mathrm{~cm}$ on the right, $2.03 \mathrm{~cm}$ on the top, and $3.05 \mathrm{~cm}$ on the bottom. Paper orientation in all pages should be in portrait style.

The existing routing technique used in wireless sensor network environment is not appropriate for mobile environment because channel needs to be changed whenever the floating node moves and there are various variables. Also, there may be a case of the floating node getting cut off as it suddenly goes beyond the limited range of net-work. Therefore, the network discussed in this study is defined as a network of fixed sensor node and floating node, and situation of getting cut off is prevented by limiting the floating node to make communication with at least one node possible.

Figure 1 is a comparison of the technique suggested in this study and the shortest hop routing. There are 2 channels from node 1 to the sink node[5]. Channel A applied a technique suggested in this study and Channel B applied the shortest hop algorithm. Equation (1) and (2) represent the two channels. $N_{i}$ signifies number i node, and $d_{x}^{i}$ represents the distance from i node to x node.

$$
\begin{gathered}
N_{1} \stackrel{d_{6}^{1}}{\longrightarrow} N_{6} \stackrel{d_{7}^{6}}{\longrightarrow} N_{7} \stackrel{d_{\text {sink }}^{7}}{\longrightarrow} N_{\text {sin } k} \\
N_{1} \stackrel{d_{2}^{1}}{\longrightarrow} N_{2} \stackrel{d_{3}^{2}}{\longrightarrow} N_{3} \stackrel{d_{4}^{3}}{\longrightarrow} N_{4} \stackrel{d_{5}^{4}}{\longrightarrow} N_{5} \stackrel{d_{\sin k}^{5}}{\longrightarrow} N_{\sin k}
\end{gathered}
$$

When transmitting a packet from node 1, channel A passes through 4 nodes and channel B passes through 2 nodes and arrives the sink node. It might appear that the total energy consumption is lesser for channel B because of fewer number of hop, but energy consumption is lesser when sending through channel A. This is possible through the output adjustment of sensor according to the distance. Theoretical explanation is as follows. The transmission radius which is basically set in node 1 is $2 \mathrm{R}$ radius. The output energy to fill $2 \mathrm{R}$ radius with electromagnetic wave is assumed to be $185 \mathrm{uJ}$. Total $555 \mathrm{uJ}$ gets consumed in the packet transmission because there are 3 outputs when applying the shortest hop algorithm. However, the use of suggested technique is as follows. $1 / 2$ range within the $2 R$ radius will be set to $R$ radius. When transmitting to nodes inside $\mathrm{R}$ radius, $23.125 \mathrm{uJ}$ of the output energy which is $1 / 8$ of $185 \mathrm{uJ}$ is enough. Therefore, $277.5 \mathrm{uJ}$ of energy which is the sum of transmission No.4 to $\mathrm{R}$ radius node and transmission No.1 to $2 \mathrm{R}$ radius node gets consumed in packet transmission. The remaining energy consumption other than the transmission energy output will be excluded. This study set the basic transmission radius of sensor to $2 R$ and suggested 2 radii by considering $1 / 2$ of distance as $R$ radius, but it can be segmented more depending on the situation. The $R$ radius node below is a node included within the $\mathrm{R}$ distance, and $2 \mathrm{R}$ radius node is more distant than $\mathrm{R}$ but is inside $2 \mathrm{R}$ distance. 


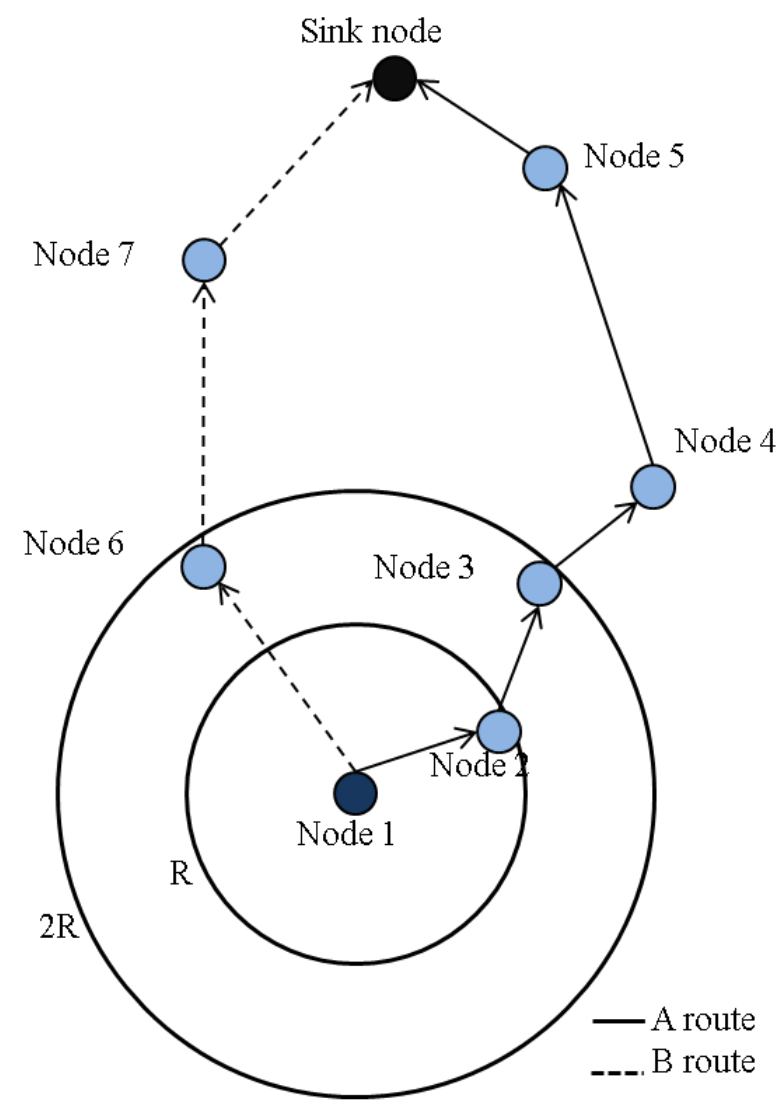

Figure 1. The Two Paths of the Node

Referred to figure 1 , the routing procedure can be mathematically explained as follows. When a sensor node is being moved, it exchanges the routing information such as nodes and location information by sending and receiving hello packet with other communicable nodes that are within coverage. Based on distance information on node 2, 3, and 6 which are within communicable range of node 1 which have data to transmit, the equation (3) is established as below.

$$
N_{1}=\left\{d_{2}^{1}, d_{3}^{1}, d_{6}^{1}\right\}
$$

$\mathrm{N} 1$ denotes the set of distance information of node 1. d denotes the distance from node 1 to node 2 , whereas d denotes the distance from node 1 to node 3 . Applying these to the sensor node, $\mathrm{n}$ of $\mathrm{N}$ can be obtained as following equation (4).

$$
\begin{gathered}
N_{1}=\left\{d_{2}^{1}, d_{3}^{1}, d_{6}^{1}\right\} \\
N_{2}=\left\{d_{1}^{2}, d_{3}^{2}, d_{4}^{2}\right\} \\
\vdots \\
N_{n}=\left\{d_{x}^{n}, d_{y}^{n}, d_{z}^{n} \ldots\right\}
\end{gathered}
$$

Once a radius $\mathrm{R}$ is set to threshold value and the equations (4) are rearranged, then the equation (5) can be obtained as follows.

$$
d_{a_{1}}^{n} \leq d_{a_{2}}^{n} \leq \cdots \leq R \leq \cdots \leq d_{a_{x}}^{n}
$$

Referring these values, distance information table is updated sequentially. To choose the next node, the sink node and distance information are used. Initially, broadcasting message sent by the sink node is used to identify the adjacent nodes to the sink node. Subsequently, in case that the sensor node is moved, location information of the sensor node can be identified since it exchanges messages with 
neighbor nodes while it moves. Additionally, the sink node sends broadcasting message periodically so that location information can be synchronized.

Figure 2 shows specific condition of network. There is a node 1 at the center. A node 2 is located at the range of a radius $R$ from the node 1 , while a node 3 is located at the range of $2 \mathrm{R}$ from the node 2 . If the proposed mechanism is applied under the assumption that the sink node is positioned at the upper side, the node 2 is selected as the next node. However, next node of the node 2 is the node 3 which exists at the $2 \mathrm{R}$ radius from the node 2 . Though the first next node is at $\mathrm{R}$ radius, the second next node exists at $2 \mathrm{R}$ radius, which means that unnecessary transmission occurs. In this case, more efficient way to transmit data is to send data from node 1 to node 3 with skipping node 2 . In routing procedure, therefore, distance information table for the selected node should be considered as well.

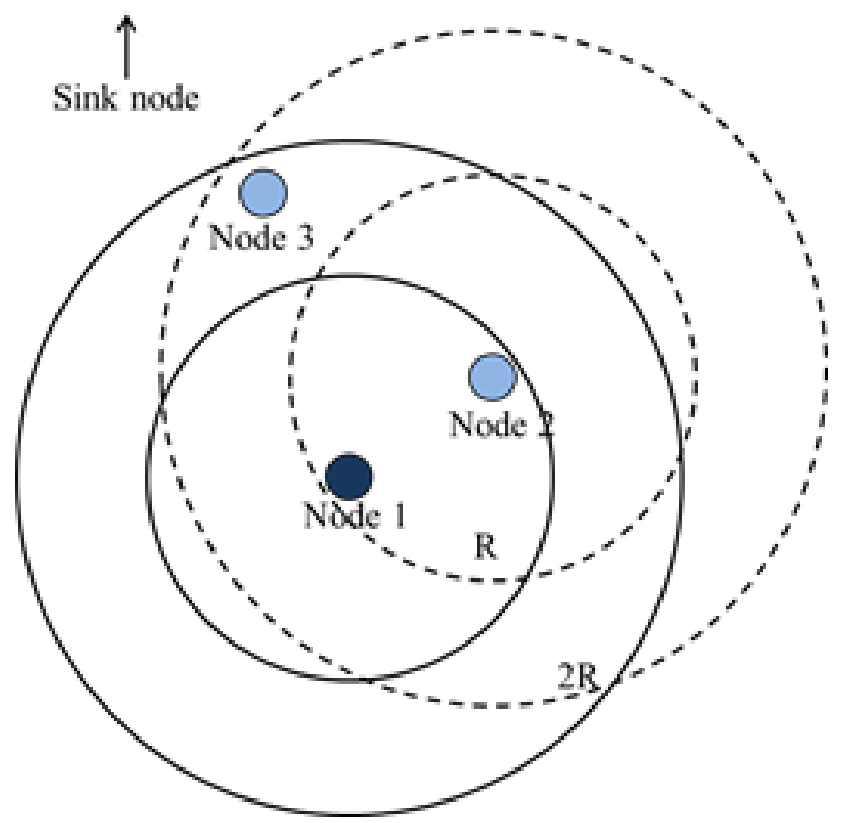

Figure 2. Condition Where Inefficient Transmission Can Occur

Figure 3 is briefly showing the routing algorithm. $N_{r}$ is the rearranged set of distance information through $\mathrm{R}$ threshold value. $N_{s}[x]$ is a value that represents the closeness of $\mathrm{x}$ node with sink node. The smaller value represents that it is closer to the sink node. The size of distance set gets allocated on i value when it starts, and traces every distance set by reducing 1 each. This study will check if $2 \mathrm{R}$ node exists because it will explore from the back, and if it exists, a node closest to the sink node will be set as a next node. Then, this study will check if node exists after setting by referring to the routing table of relevant node in $\mathrm{R}$ node. If $2 \mathrm{R}$ node does not exist, a node closest to the sink node in $\mathrm{R}$ node will be set as the next node. 


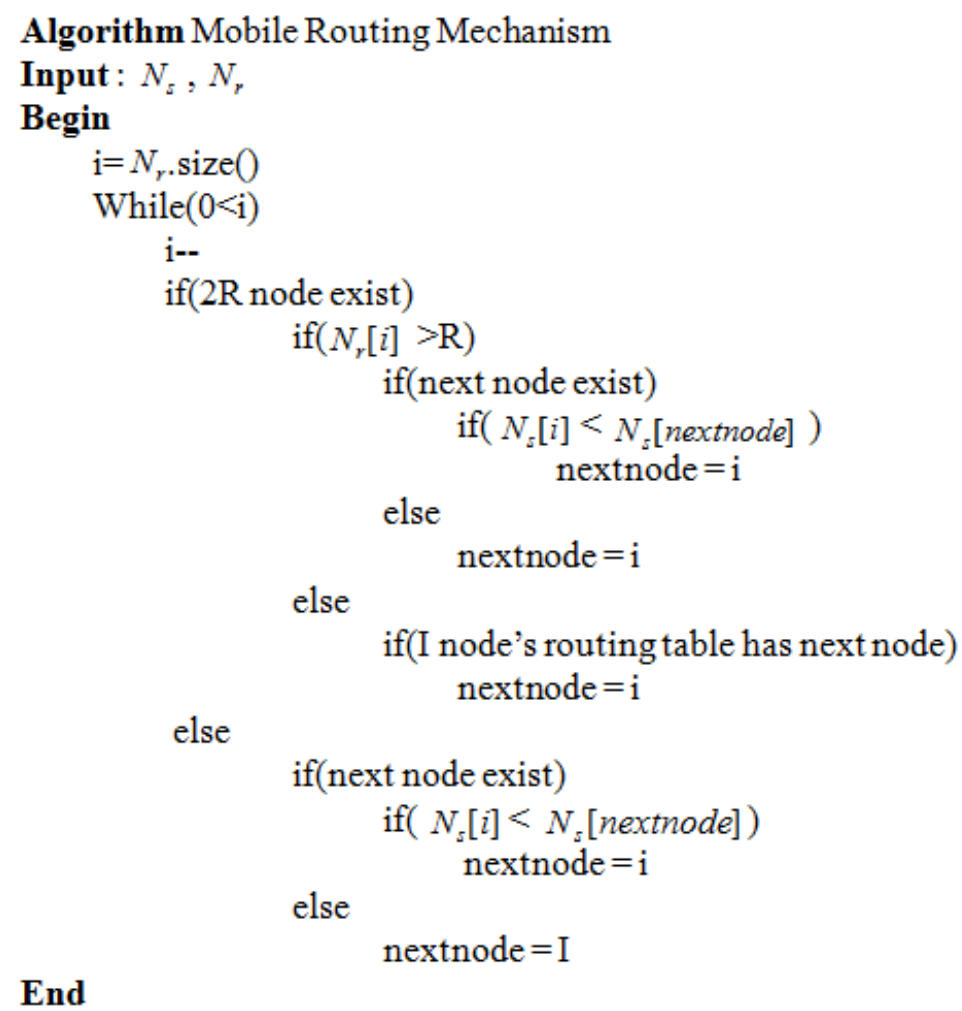

Figure 3. The Proposed Routing Algorithm

\section{Performance Evaluation and Analysis}

\subsection{Simulation Configuration}

The network architecture used for simulation is as shown in figure 4. A source node is a node which generates packets for obtaining simulation results. A sink node is a destination node which eventually receives all the packets in the network. A background traffic node is a node which creates packets periodically at a certain interval so as to cause congestion in the network. The rest of the nodes are relay nodes which are divided into two categories: fixed node and mobile node. Simulation can be run in fixed-time mode and life-cycle mode. In fixed-time mode, simulation software outputs the information of packets which arrive at the sink node on completion of execution during the fixed time period. In life-cycle mode, the simulation output comes out when a node with depleted energy occurs for the first time among the nodes existing in the network. Packet generation period for source node and background traffic node can be set prior to the simulation. It is assumed that each node identifies the closer node to the sink node by receiving broadcast message sent by the sink node initially. Each node maintains $\mathrm{R}$ node table and $2 \mathrm{R}$ node table. During simulation, mobile nodes look for directions to which they are able to move among up, down, left and right once every fixed period of time. Then they randomly move to one of available directions. Consequently, routing table in each node will be updated whenever mobile node moves. 


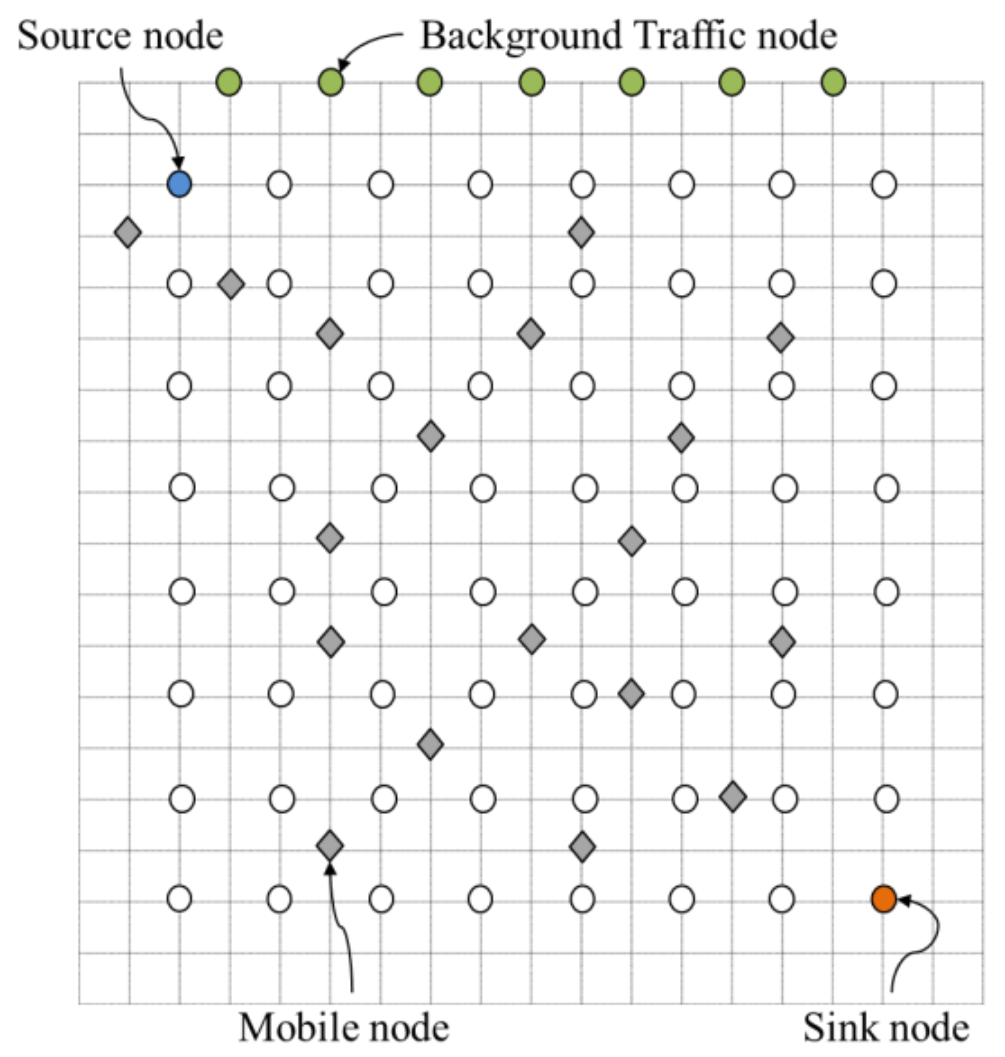

Figure 4. The Proposed Network Architecture

\subsection{Simulation Environment Setting}

Table 1 shows the values of environment variables set for the simulation. The network is constructed using 64 fixed nodes and 30 mobile nodes. Those nodes are randomly placed on $19 \times 19$ grid structure. Among fixed nodes, the leftmost and topmost node is set to be a source node, while the rightmost and bottommost node is set to be a sink node. Background traffic nodes are placed on the grid in order to set network congestion. Transmission energy is set differently for the case that packet is sent out in radius $R$ and the case in radius $2 R$. The proposed routing is proceeded in the steps as follows. Firstly, if 2R-radius nodes exist, the closest node to the sink node and the second closest node among the $2 \mathrm{R}$-radius nodes are selected. Then the routing algorithm checks if any $\mathrm{R}$-radius node exists. If not found, packet will be transmitted to one of the selected nodes which is closest and possesses highest energy. If R-radius node is found, it will check if R-radius routing table of the node contains the selected $2 \mathrm{R}$ node. If there is not, the packet will be sent out to the selected $2 \mathrm{R}$ node. If there is, the packet will be sent out to the found node.

Table 1. Simulation Environment Variables

\begin{tabular}{c|c}
\hline \hline Parameter & Value \\
\hline \hline Network size & $(0,0) \sim(360,360)$ \\
Sink node & $(320,320)$ \\
Source node & $(40,40)$ \\
Background traffic node & $(3,3) \sim(15,15)$ (only \\
odd number) \\
The number of fixed nodes
\end{tabular}




\begin{tabular}{c|c} 
The number of mobile nodes & 30 \\
Transmission range & $\mathrm{R}, 2 \mathrm{R}$ \\
Initial energy of node & $1 \mathrm{~J}$ \\
Energy consumption for R-radius & $46 \mathrm{uJ}$ \\
transmission & \\
Energy consumption for 2R-radius & $185 \mathrm{uJ}$ \\
transmission & $83 \mathrm{uJ}$ \\
Energy consumption when receiving & $64 \mathrm{uJ}$ \\
Energy consumption in idle & $100 \mathrm{~ms}$ \\
\hline Simulation period of time &
\end{tabular}

\subsection{Analysis of Simulation Results}

For performance evaluation, we compare the proposed mechanism to the conventional minimum hop routing method in terms of the average packet latency, average energy consumption and network life time. Simulation was carried out by varying packet generation interval for source node and background traffic node and varying traffic congestion ranging from $10 \%$ to $80 \%$. Figure 5, 6 and 7 show the graphs from the simulation results.

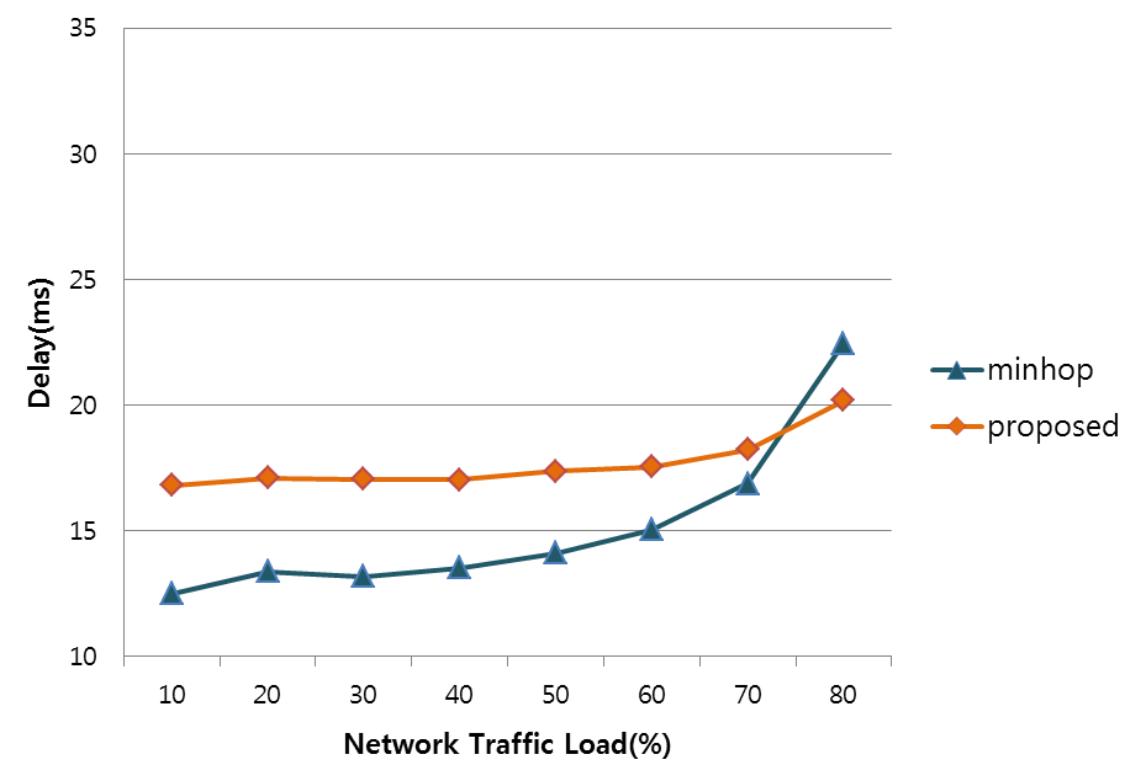

Figure 5. Graph of Average Packet Latency 


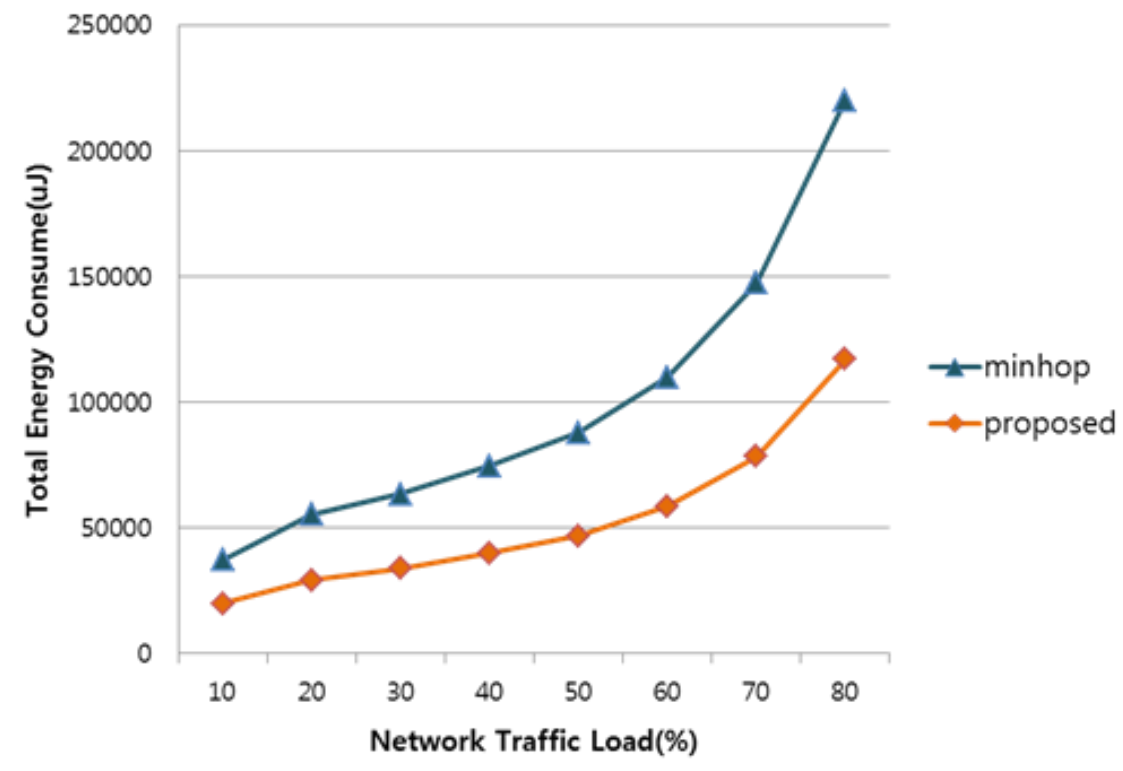

Figure 6. Graph of Average Energy Consumption

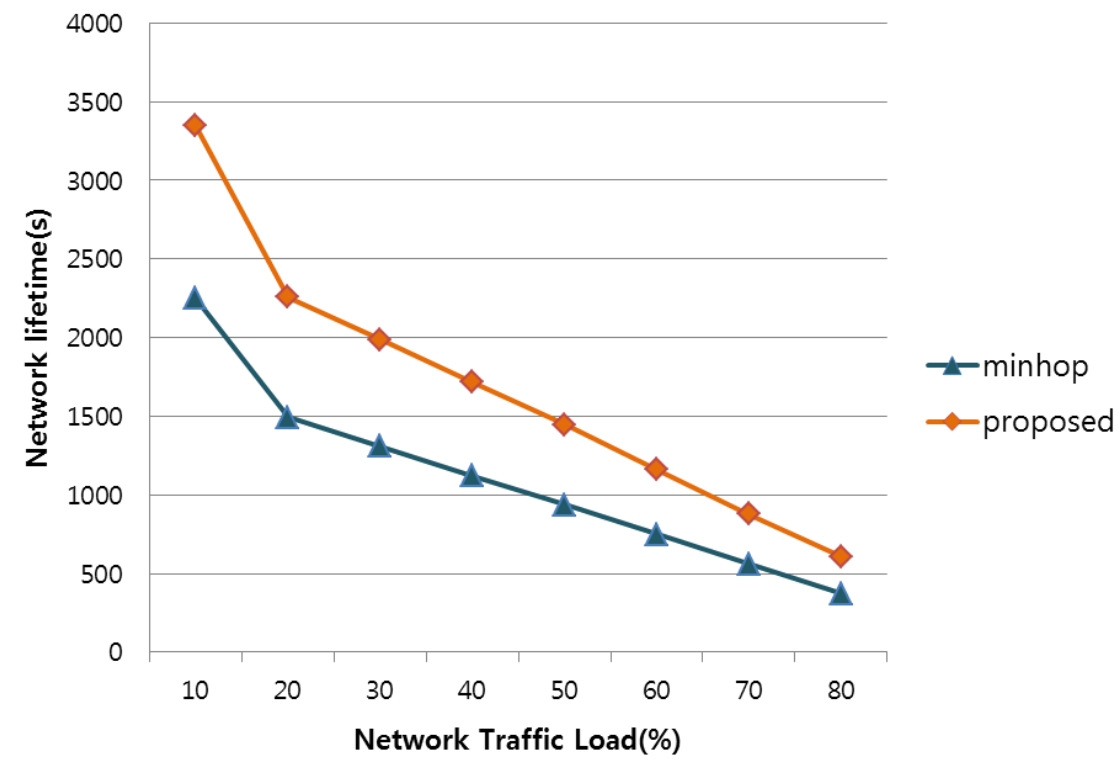

Figure 7. Graph of Network Lifespan

Figure 5 shows average latency time of packets. The average number of hops is measured to be 12 and 17 for the minimum hop algorithm and the proposed mechanism respectively. The difference is 5 hops which corresponds to approximately $4.8 \mathrm{~ms}$ of transmission time if the transmission time is assumed to be $0.96 \mathrm{~ms}$. However, as the degree of network congestion gets higher, difference of transmission time gets decreased. At $80 \%$ of congestion degree, the time taken for packet transmission of the proposed mechanism $(20.18 \mathrm{~ms})$ even goes down below that of minimum hop algorithm $(22.46 \mathrm{~ms})$. When network gets crowded more, it is more likely to wait for availability of neighbour node because the neighbour node is frequently busy in sending or receiving packets. In this case, the proposed mechanism shows better performance since it sends out the packet within $\mathrm{R}$ radius, which eventually reduces the number of neighbour nodes to check. Figure 6 is a 
graph to show average energy consumption. At $80 \%$ congestion degree, the sink node receives total 100 packets with $46 \%$ reduced energy consumption. Figure 7 shows network lifespan. At 10\% congestion degree, the lifetime is measured to 3346 seconds and 2250 seconds for the proposed mechanism and the minimum hop algorithm respectively. As the energy consumption level gets lower, the saved amount of energy contributes to the prolonged lifespan of network. Simulation results indicate that routing method of the proposed mechanism is superior to conventional method in terms of energy efficiency, especially under specific circumstance.

\section{Conclusion}

This paper proposes the adaptive transmission mechanism which enables to adjust transmission energy with consideration of distance between nodes and routing data in mobile sensor network. Since the communication between wireless sensor nodes is achieved by electromagnetic waves, two distant nodes can communicate only if the electromagnetic waves sent out by one node are strong enough to be propagated further to the counter node. Energy required for wireless communication depends on the distance between two nodes. In general, the amount of energy consumption for radio communication is proportional to the square or the fourth power of the distance. In this paper, we propose an enhanced mechanism which determines the subsequent node in accordance to the distance to adjacent nodes and enables to properly control the transmission output level corresponding to the distance to the chosen node under the circumstance that sensor node passes packets destined to the sink node. Performance of the proposed mechanism has been simulated. According to the simulation results, average number of hops is 12 and 17 for conventional minimum hop algorithm and the proposed mechanism respectively. Since transmission time is set to $0.96 \mathrm{~ms}, 5$ hops difference corresponds to five times of $0.96 \mathrm{~ms}$ which equals to $4.8 \mathrm{~ms}$. However, the simulation results indicate that the time difference gets smaller as the degree of network congestion gets higher. It can be explained that the proposed mechanism produces more stable performance in terms of average packet latency as more traffic takes place. In terms of average energy consumption, the proposed mechanism saves approximately $46 \%$ of energy consumed by the conventional method. In respect to network lifespan, the proposed mechanism prolongs the network lifetime longer than the conventional minimum hop algorithm by up to $33 \%$ at $10 \%$ of congestion degree.

Thus it is demonstrated that the mechanism proposed in this paper outperforms the conventional method which produces fixed level of transmission output.

\section{Acknowledgments}

"This research was supported by the MSIP(Ministry of Science, ICT and Future Planning), Korea, under the ITRC(Information Technology Research Center) support program (IITP-2016-H8601-16-1007) supervised by the IITP(Institute for Information \& communications Technology Promotion)"

\section{References}

[1] I. F. Akyildiz, T. Melodia and K. R. Chowdhury, "A survey on wireless multimedia sensor networks", The Journal of Computer Networks, vol. 51, no. 4, (2007). pp. 921-960.

[2] J. Yong-Jea, B. Si-Yeong and L. Sung-Keun, “An Energy Efficiency Routing Algorithm in Wireless Sensor Networks", Future Generation Information Technology Lecture Notes in Computer Science, vol. 7105, (2011). pp. 183-189.

[3] M. Di Francesco, S. K. Das, and G. Anastasi, "Data collection in wireless sensor networks with mobile elements: A Survey”, ACM Trans. Sensor Networks, vol.8 no. 1, (2011). pp. 1-31. 
[4] R. Jaichandran, A. Irudhayaraj, and J. Raja, "Effective strategies and optimal solutions for hot spot problem in WSNs", in Information Sciences Signal Processing and their Applications (ISSPA), 2010 10th Int. Conf. on, (2010). pp. $389-392$.

[5] W. Liang, J. Luo and X. Xu, "Prolonging network lifetime vis a controlled mobile sink in WSN", in Global Telecommunications Conf. (GLOBECOM 2010), IEEE, (2010). pp. 1-6.

[6] C. E. Larsen, R. Trip and C. R. Johnson, "Methods for procedures related to the electrophysiology of the heart", U.S. Patent 5,529,067, (1995)

\section{Authors}

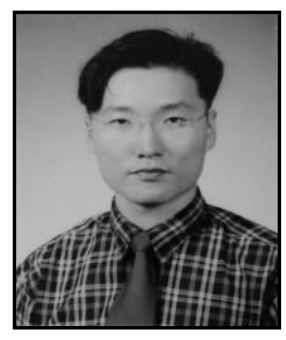

Jun-Hyoung Kim, he received the B.S. degrees in mathematics from Korea University, Seoul, Korea, in 1998, and the M.S. student in multimedia engineering at the Sunchon National University, Suncheon, Korea, since 2013. His current research interests are wireless sensor network, multimedia communication and Internet QoS.

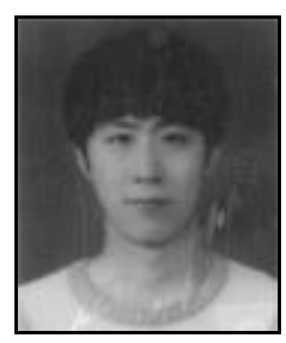

Jeong-Hyeon Park, he received the B.S. degrees in multimedia engineering from Sunchon National University, Suncheon, Korea, in 2014, and the M.S. student in multimedia engineering at the Sunchon National University, Suncheon, Korea, since 2014. His current research interests are wireless sensor network, multimedia communication and Internet QoS.

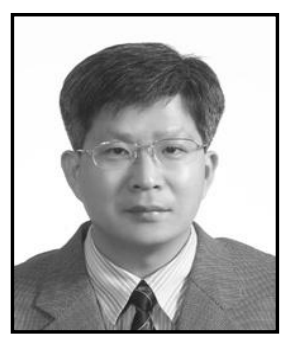

Sung-Keun Lee, he received the B.S., M.S., and Ph. D. degrees in electronics engineering from Korea University, Seoul, Korea, in 1985, 1987, and 1995, respectively. From 1987 to 1992, he was with Samsung electronics Co., Ltd, Korea. He joined the department of Multimedia Engineering, Sunchon National University, Suncheon, Korea, in 1997, where he is currently a Professor. His research interests include IoT, wireless sensor network, multimedia communication, energy efficient Ethernet, and Internet QoS. 